\title{
Acknowledgement of Reviewers
}

We gratefully acknowledge the work of reviewers for Journal of Plant Diseases and Protection. The standards of scientific publishing are upheld by peer review and the work of our reviewers. We thank the many scientists who have given of their time and expertise to offer their judgements and to help authors in improving manuscripts during the year 2012.

Rene van Acker, Guelph, Canada

Liv Åkerblom Espeby, Uppsala, Sweden

Gerhard Adam, Wien, Austria

Gabriele Berg, Graz, Austria

Marco Beyer, Belvaux, Luxembourg

Regina Belz, Hohenheim, Germany

Thomas Brand, Oldenburg, Germany

Patrick Brunner, Zürich, Switzerland

Frank Burghause, Bad Kreuznach, Germany

Eric Cascales, Marseille, France

Mirna Ćurković-Perica, Zagreb, Croatia

Amer Dababat, Ankara, Turkey

Danièle Debieu, Thiverval-Grignon, France

Jane Debode, Merelbeke, Belgium

Francois Delmotte, Bordeaux, France

Evgenia Dor, Ramat Yishay, Israel

Hanna Dorna, Poznan, Poland

Antonin Dreiseitl, Kroměříž, Czech Rep

Hans G Drobny, Neu Isenburg, Germany

Barbara Edler, Göttingen, Germany

Xavier Foissac, Bordeaux, France

Lorenzo Furlan, Padua, Italy

Luciana Galetto, Torino, Italy

Jacques Gasquez, Dijon, France

Friedhelm Gauhl, Vienna, Austria

Raul NC Guedes, Vicosa, Brazil

John Hammond, Beltsville, USA

Stephan Hartmann, Freising, Germany

Udo Heimbach, Braunschweig, Germany

Annette Herz, Darmstadt, Germany

Monika Heupel, Bonn, Germany

Christian Hillnhütter, Bonn, Germany

Bernd Holtschulte, Einbeck, Germany

Bernd Hommel, Kleinmachnow, Germany

Don Huber, West Lafayette, USA

Ebrahim Izadi, Mashhad, Iran

Heikki Jalli, Jokioinen, Finland

Wolfgang Jarausch, Neustadt, Weinstr., Germany

Branka Javornik, Ljubljana, Slovenia

Hanns-Heinz Kassemeyer, Freiburg, Germany

Walter K Kast, Weinsberg, Germany

Polona Kogovsek, Ljubljana, Slovenia
Andreas Kollar, Dossenheim, Germany

Hansjoerg Krähmer, Frankfurt, Germany

Kristian Kristensen, Tjele, Denmark

Stefan Kunz, Konstanz, Germany

David Legland, Thiverval-Grignon, France

Frank J Louws, Raleigh, North Carolina, USA

Matthias P Lutz, Wädenswil, Switzerland

Christine Martin, Paris, France

Marta Martini, Udine, Italy

Wulf Menzel, Braunschweig, Germany

Berthold Metzler, Freiburg, Germany

Volker Mohler, Freising, Germany

Jan Nechwatal, Freising, Germany

Lescek Orlikowski, Skierniewice, Poland

William A Overholt, Gainesville, USA

Olivier Parisi, Gembloux, Belgium

Jan Petersen, Bingen, Germany

Maryam Rafiqi, Gießen, Germany

Emilie E Regnier, Columbus, USA

Marion Röder, Gatersleben, Germany

Diego Rubiales, Córdoba, Spain

Sabrina Sarrocco, Pisa, Italy

Maurizio Sattin, Legnaro, Italy

Harald Scherm, Athens, USA

Pieter Spanoghe, Ghent, Belgium

Otmar Spring, Stuttgart, Germany

Eva Stukenbrock, Marburg, Germany

Brett Summerell, Sydney, Australia

Sotirios Tjamo, Athens, Greece

Thomas Thieme, Sagerheide, Germany

Tim C Thoden, Freiburg, Germany

Federico Tinivella, Torino, Italy

Jozef Vanden Broeck, Leuven, Belgium

Mark Varrelmann, Göttingen, Germany

Ferenc Viranyi, Gödöllő, Hungary

Roland Weber, Jork, Germany

Rommie van der Weide, Wageningen, Netherlands

Andreas Westphal, Braunschweig, Germany

Kris AG Wyckhuys, Hanoi, Vietnam

Ye Xia, Lexington, KY, USA

Christin Zachow, Graz, Austria

Volker Zahn, Hannover, Germany 